\title{
Habitual foot strike pattern does not affect simulated Triceps Surae muscle metabolic
}

8 Running title: Muscle energy between foot strike types

9 Keywords: dynamic optimization, forefoot strike, Gastrocnemius medialis, rearfoot strike, Soleus, 10 ultrasound

11 Summary statement: Simulated Triceps Surae muscle or whole body metabolic energy consumption 12 is not different between mid-/forefoot and rearfoot strikers.

13

14 List of symbols and abbreviations:

15 ANOVA Analysis of variance

16 AT

$17 E \quad$ Metabolic energy consumption rate

18 EMG Electromyography

19 FF Mid-/forefoot strike

$20 \quad \mathrm{GL} \quad$ Gastrocnemius lateralis

21 GM Gastrocnemius medialis

$22 H_{A} \quad$ Activation heat rate

$23 H_{M} \quad$ Maintenance heat rate

$24 H_{S L} \quad$ Shortening/lengthening heat rate

25 MTU Muscle tendon unit

26 RF Rearfoot strike

27 SEE Series elastic element

28 SOL Soleus

29 TS Triceps Surae

$30 \quad W \quad$ Mechanical work rate 


\section{$\underline{\text { Abstract }}$}

34 Foot strike pattern affects ankle joint work and Triceps Surae muscle-tendon dynamics during running. Whether these changes in muscle-tendon dynamics also affect Triceps Surae muscle energy consumption is still unknown. In addition, as the Triceps Surae muscle accounts for a substantial amount of the whole body metabolic energy consumption, changes in Triceps Surae energy consumption may affect whole body metabolic energy consumption. However, direct measurements of muscle metabolic energy consumption during dynamic movements is hard. Model-based approaches can be used to estimate individual muscle and whole body metabolic energy consumption based on Hill type muscle models. In this study, we use an integrated experimental and dynamic optimization approach to compute muscle states (muscle forces, lengths, velocities, excitations and activations) of 10 habitual mid-/forefoot striking and 9 habitual rearfoot striking runners while running at 10 and $14 \mathrm{~km} / \mathrm{h}$. The Achilles tendon stiffness of the musculoskeletal model was adapted to fit experimental ultrasound data of the Gastrocnemius medialis muscle during ground contact. Next, we calculated Triceps Surae muscle and whole body metabolic energy consumption using four different metabolic energy models provided in literature. Neither Triceps Surae metabolic energy consumption $(p>0.35)$, nor whole body metabolic energy consumption $(p>0.14)$ was different between foot strike patterns, regardless of the energy model used or running speed tested. Our results provide new evidence that mid-/forefoot and rearfoot strike pattern are metabolically equivalent.

\section{Introduction}

52 The metabolic energy consumed during submaximal running, often referred to as running economy, is 53 an important factor determining endurance running performance (Jones and Carter, 2000). Reduced 54 energy consumption corresponds to improved running economy and hence superior endurance 55 performance (Hoogkamer et al., 2016; Kipp et al., 2019). As such, researchers try to identify running 56 patterns with minimal metabolic energy consumption (Moore, 2016). One aspect of people's running 57 pattern is foot strike pattern. Although foot strike pattern is a continuum, generally three different 58 foot strike patterns are considered: forefoot strike, midfoot strike and rearfoot strike (Cavanagh and Lafortune, 1980).

60 While rearfoot striking is the most common running pattern during shod running (Hasegawa et al.,

61 2007; Kasmer et al., 2013; Larson et al., 2011), there seems to be a widespread popular belief that 62 forefoot striking would be more economical than rearfoot striking. This belief is based upon evidence 63 that a greater percentage of mid-/forefoot strikers appear among the first finishers in long distance 
races (de Almeida et al., 2015; Hasegawa et al., 2007), suggesting forefoot striking to be more economical. However, studies comparing metabolic energy consumption between habitual forefoot and habitual rearfoot strikers found no difference in whole body metabolic energy consumption (Gruber et al., 2013) or even lower energy consumption in rearfoot strikers compared to their forefoot striking colleagues at 11 and $13 \mathrm{~km} / \mathrm{h}$ but not at $15 \mathrm{~km} / \mathrm{h}$ (Ogueta-Alday et al., 2014).

Available analyses of the kinetic and kinematic differences between foot strike patterns do not clearly provide evidence for either differences in or unchanged energy consumption with foot strike patterns. Differences in foot strike patterns induce changes in negative ankle work with forefoot striking demonstrating greater negative ankle work (Stearne et al., 2014). This ankle work is predominantly absorbed by the muscle-tendon unit (MTU) spanning the ankle joint, i.e., Triceps Surae muscle and the in series connected tendinous tissue (SEE, series elastic element). Hence, differences in ankle work may affect the MTU and subsequently the energy consumption of this Triceps Surae muscle. We recently demonstrated that during early stance one of the Triceps Surae muscles, the Gastrocnemius medialis (GM), produces greater muscle force at lower contraction velocity in mid-/forefoot strikers compared to rearfoot strikers. Higher muscle force production suggests more muscle activation and thus higher metabolic energy consumption, whereas lower contraction velocities appear to be more force efficient and would therefore reduce muscle activation and thus metabolic energy consumption (Hill, 1922; van der Zee, Lemaire and van Soest, 2019). Hence, we hypothesized that the differences in metabolic energy consumption would counteract each other and no difference in GM metabolic energy consumption would exist (Swinnen et al., 2019). While it is still unknown whether foot strike pattern induces differences in Gastrocnemius lateralis (GL) and Soleus (SOL), the other two Triceps Surae muscles, previous research estimated that the Triceps Surae accounts for 25 to $40 \%$ of the whole body metabolic energy consumption (Fletcher and Maclntosh, 2017). Consequently, if Triceps Surae muscle metabolic energy consumption is similar between foot strike patterns, we would expect no differences in whole body metabolic energy consumption between foot strike patterns either.

Model-based approaches have been used to estimate individual muscle and whole body metabolic energy consumption based on Hill type muscle models (Bhargava et al., 2004; Miller, 2014; Uchida et al., 2016; Umberger, 2010; Umberger et al., 2003). However, to obtain reliable simulation results, a close match between simulated and experimental data is essential. Here, we used experimental dynamics ultrasound data from the GM to improve our dynamic optimization and as such, ensure more reliable estimations of muscle metabolic energy consumption. We used four different metabolic energy models (Bhargava et al., 2004; Uchida et al., 2016; Umberger, 2010; Umberger et al., 2003) to calculate Triceps Surae muscle and whole body metabolic energy consumption of habitual mid/forefoot and rearfoot strikers running at 10 and $14 \mathrm{~km} / \mathrm{h}$. We hypothesized that neither Triceps Surae 
Table 1 Participants demographics. All data are expressed as mean $\pm S D$. No significant difference between groups.

\begin{tabular}{lcc}
\hline & Forefoot strike & Rearfoot strike \\
\hline Body mass (kg) & $65.2 \pm 7.7$ & $72.7 \pm 12.5$ \\
\hline Body height (m) & $1.78 \pm 0.07$ & $1.81 \pm 0.08$ \\
\hline $\begin{array}{c}\text { Training volume } \\
\text { (km/week) }\end{array}$ & $53 \pm 23$ & $49 \pm 15$ \\
\hline
\end{tabular}

nor whole body metabolic energy consumption would be different between foot strike patterns. A secondary aim is to assess whether these findings are robust to the metabolic energy models used.

\section{Methods}

Participants. Ten habitual mid-/forefoot strikers (6 males, 4 females) and nine habitual rearfoot strikers (6 males, 3 females) participated in this study (Table 1). All participants were regular runners with at least 2 years running experience, running $30 \mathrm{~km} /$ week or more and were able to run $10 \mathrm{~km}$ within 45 minutes. They did not have any Achilles tendon or calf injury in the last six months and had no prior Achilles tendon surgery. Written informed consent, approved by the local ethical committee (Medical Ethical Committee of UZ Leuven), was obtained at the start of the experiment.
108

Experimental procedure. The experimental procedures have been described in detail in our earlier publication on gastrocnemius medialis muscle-tendon interaction and muscle force production in this group of runners (Swinnen et al., 2019). Briefly, after a 10 minutes warm-up, participants ran 5 minutes on a force measuring treadmill (Motekforce Link, Amsterdam, The Netherlands): 2.5 minutes at 10 and at $14 \mathrm{~km} / \mathrm{h}$, in randomized order. We collected kinetic, kinematic, muscle activation and ultrasound data of at least four strides during the last minute of each running speed. All measurements were synchronized through a trigger pulse signal sent from the ultrasound device.

Kinetics, kinematic and foot strike angle. Thirteen infrared cameras (Vicon, Oxford Metrics, UK) captured the motion of an extended plug-in-gait marker set (forty-seven reflective markers, Fig. S1) at a sampling frequency of $150 \mathrm{~Hz}$. We used OpenSim 3.3 (OpenSim, Stanford, USA) to first scale the Hamner musculoskeletal model based on the subject's dimensions (Hamner et al., 2010) and to subsequently compute joint kinematics using a Kalman smoothing algorithm (De Groote et al., 2008). Muscle tendon unit lengths were calculated using OpenSim's Muscle Analysis Tool. 
122 Ground reaction force data, sampled at $900 \mathrm{~Hz}$, was first low pass filtered with a cut-off frequency of

$12320 \mathrm{~Hz}$ and used to determine ground contact phase adopting a $30 \mathrm{~N}$ threshold. We determined foot 124 strike angle using a marker based method (Altman and Davis, 2012). At initial ground contact, we drew 125 a line through the first metatarsal-phalangeal joint marker and heel marker of the left foot. The angle 126 between this line and the ground was calculated and considered as the foot strike angle. Following 127 Altman and Davis (2012) runners with a foot strike angle greater than $8^{\circ}$ were considered rearfoot 128 strikers, while runners with a foot strike angle under $8^{\circ}$ were considered mid-/forefoot strikers. Foot 129 strike angle was averaged over the strides used for ultrasound analysis. Foot strike type (rearfoot or 130 mid-/forefoot) was consistent within subjects across running speeds.

131 We calculated joint torques using OpenSim's Inverse Dynamics Tool based on joint kinematics and 132 ground reaction forces. Joint torques were low pass filtered using a recursive fourth order Butterworth 133 filter with cut-off frequency of $20 \mathrm{~Hz}$.

134 Dynamic ultrasound imaging. We collected dynamic ultrasound images of the GM muscle 135 fascicles of the left leg with a B-mode ultrasound system (Telemed Echoblaster 128 CEXT system) 136 sampling at $86 \mathrm{~Hz}$. The linear transducer (UAB Telemed, Vilnius, Lithuania, LV 7.5/60/128Z-2) was 137 placed on the mid-belly of the muscle, aligned with the muscle fascicles and attached to the calf with 138 tape and bandages. To analyze the GM muscle fascicle lengths and pennation angles we used a semi139 automatic tracking algorithm (Farris and Lichtwark, 2016). We analyzed at least four strides and 140 calculated fascicle length changes relative to fascicle length at toe-off. All data were splined to 100 141 data points per ground contact, starting at initial contact.

142 Muscle activity. We used surface electromyography (EMG) to determine GM and SOL muscle 143 activity of the right leg through a wireless EMG acquisition system (ZeroWire EMG Aurion, Milano, 144 Italy) measuring at $900 \mathrm{~Hz}$. EMG signals were first band-pass filtered (20-400 Hz), rectified and low145 pass filtered $(20 \mathrm{~Hz})$. For each subject and muscle, EMG waveforms were normalized to maximal 146 activation, determined as the maximal activation of each muscle using a moving average over 10 data 147 points. Due to technical issues, the EMG data of the GM of one participant (mid-/forefoot striker) and 148 SOL of three participants (2 mid-/forefoot strikers and 1 rearfoot striker) could not be used.

149 Comparison between experimental EMG and simulated activation of the GM and SOL demonstrated 150 similar trends, yet due to our optimization criteria (minimization of muscle activation squared) pre151 activation is not predicted (Fig. S2).

152 Estimating muscle and whole body metabolic energy consumption. Several models for 153 estimating muscle metabolic energy rate have been proposed and it is yet unclear which model yields 154 the most valid results. We, therefore, used multiple models primarily to assure that our results are 
155 independent from the metabolic energy model used. Our goal was not to compare the different energy

156 models as we do not have experimental data of the whole body metabolic energy consumption for 157 comparison (for comparison between metabolic energy models see Miller 2014 and Koelewijn et al., 158 2019). All models required the muscle states (i.e., muscle activations, excitations, lengths, velocities 159 and forces) as inputs. To obtain these muscle states we solved the muscle redundancy problem using 160 a dynamic optimization algorithm that takes into account muscle-tendon dynamics (i.e., muscle 161 activation and contraction dynamics) of the 43 lower limb muscles of the left leg in our model (De 162 Groote et al., 2009; De Groote et al., 2016). Individual muscle moment arms, muscle tendon unit 163 lengths and muscle properties were extracted from the scaled OpenSim model and were input to the 164 muscle redundancy solver. We scaled maximal isometric muscle force based on the subject's body 165 mass and height (Handsfield et al., 2014). To avoid maximal muscle activations and unrealistically high 166 reserve actuator forces, muscle forces were multiplied by 3 for all participants. The Triceps Surae 167 muscles, containing the GM, GL and SOL, were modeled as three separate muscle-tendon units, with 168 the tendon representing the Achilles tendon. To ensure a close match between experimental GM 169 muscle fascicle length changes and simulated GM muscle fascicle lengths, we adjusted the normalized 170 tendon stiffness, a scaling factor to calculate $\mathrm{GM}, \mathrm{GL}, \mathrm{SOL}$ tendon stiffness based on the ratio between 171 maximal isometric force and tendon slack length, to a value of 5 for all participants. As such, average 172 contraction velocity $(p>0.24)$ nor contraction range during ground contact $(p>0.19)$ of our simulated 173 GM muscle fascicle length changes were different from our experimental muscle fascicle length 174 changes (Figure 1). Gerus et al. (2015) previously stated that the Achilles tendon is more compliant 175 than the generic tendon stiffness as described by Zajac (1989). We tested multiple other values 176 (ranging from 4 to 35 ) where 5 gave the best match. The normalized stiffness for all other muscles was 177 kept on the default value of 35. Joint torques served as inputs to solve the muscle redundancy problem 178 by minimizing the squared muscle activation. We solved the dynamic optimization problem through 179 direct collocation using GPOP-II software (Patterson and Rao, 2014). Subsequently the resulting 180 nonlinear equations was solved using ipopt (Wächter and Biegler, 2006). In 9 out of the 154 ground 181 contacts analyzed the optimization algorithm failed to find an optimal solution, these strides were 182 excluded 

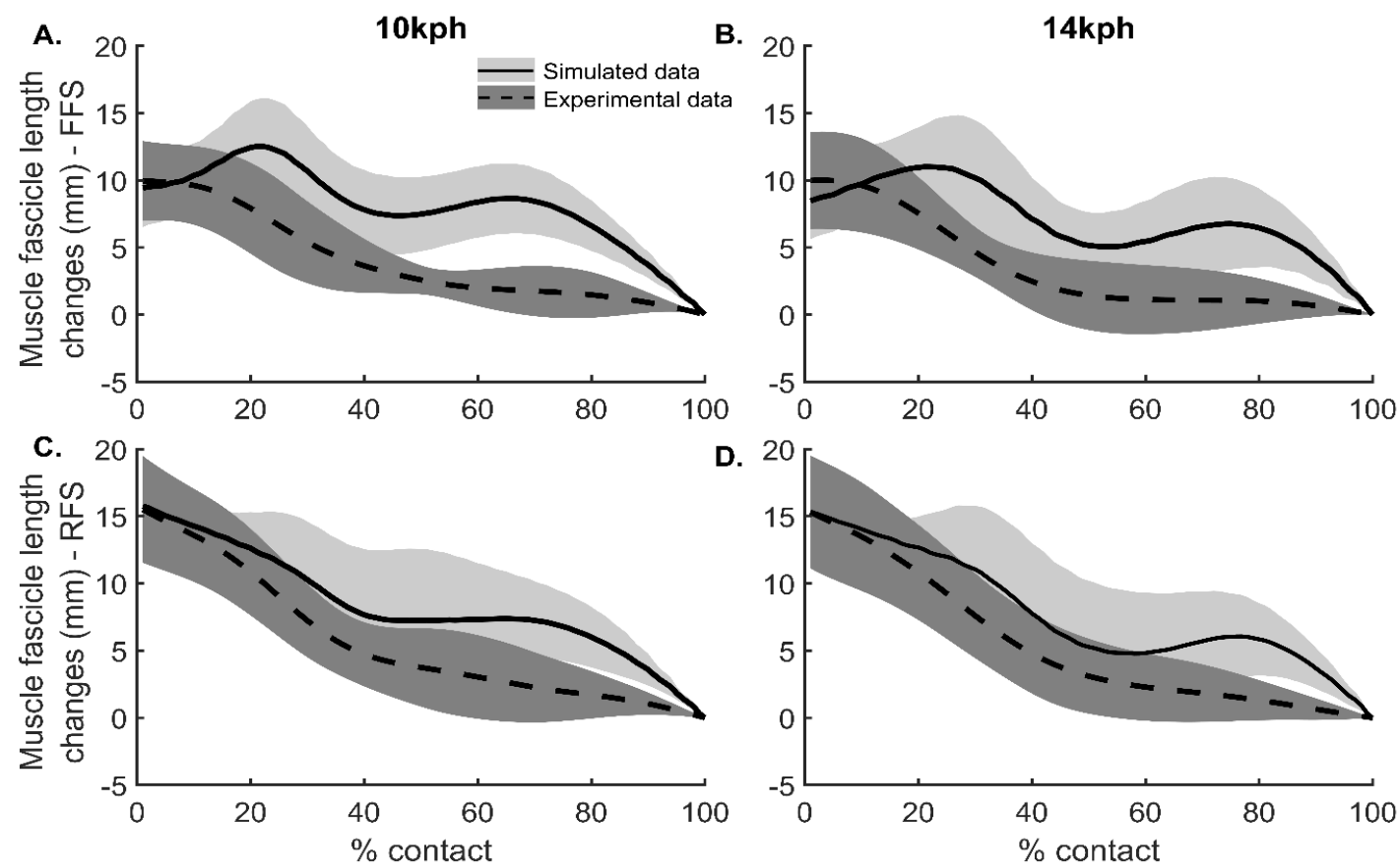

Figure 1. Simulated (solid) and experimental (dashed) GM muscle fascicle length changes during ground contact in mid/forefoot strikers $(A, B ; n=10)$ and rearfoot strikers $(C, D ; n=9)$ at $10 \mathrm{~km} / \mathrm{h}(A, C)$ and $14 \mathrm{~km} / \mathrm{h}(B, D)$. Muscle fascicle length changes are normalized to muscle fascicle length at toe off. Shaded area represent standard deviation.

Next, the simulated muscle states were used as input in four models to estimate muscle metabolic energy rate $E$ that are consistent with Hill based muscle dynamics: Umberger, Gerritsen and Martin (2003) (U03), Bhargava, Pandy and Anderson (2004) (B04), Umberger (2010) (U10) and Uchida et al. (2016) (U16). All these models had the same general form to calculate energy expenditure:

$$
E=H_{A}+H_{M}+H_{S L}+c W
$$

Where $H_{A}, H_{M}$ and $H_{S L}$ are the heat production rates of the muscles for activation, maintenance and shortening/lengthening respectively, $W$ is the muscle mechanical work rate where concentric work is defined positively and $C$ is weighting factor depending on the type of work (concentric of eccentric). Major differences between the models are how they treat eccentric muscle work and how they weight muscle lengthening heat rate. While in $\mathrm{U} 03$ and $\mathrm{U} 16$ negative mechanical work (i.e., metabolic energy generation) is incorporated, B04 and $\mathrm{U} 10$ are restricted to positive mechanical work only, negative mechanical work is excluded, and the lengthening heat rate coefficient is adapted. Apart from these differences, the heat rate calculations have similar terms between the models, though the scaling factors used are different. Activation and maintenance heat rates are generally defined by muscle mass/force, length and fiber type composition while shortening/lengthening heat rate depend on muscle contraction velocity. U03, U10 and U16 scale these heat rates by muscle activation whereas B04 does not. We refer to the specific papers for more detailed information on the models. 
204 Muscle metabolic energy rate was integrated over time to obtain metabolic energy consumption 205 during one stance phase which was then multiplied by 2 , to account for both legs, and multiplied by 206 the stride frequency to obtain metabolic energy rate in Watts. The metabolic energy consumed by the 207 Triceps Surae muscles was normalized to their respective muscle mass. We computed whole body metabolic energy expenditure as the sum of metabolic energy consumed by all 43 muscles included in the model and added a basal rate of $1.2 \mathrm{~W} / \mathrm{kg}$ (Waters and Mulroy, 1999). Whole body metabolic energy consumption was normalized to body mass.

Statistics. All data are presented as mean \pm standard deviation. We categorized our data in 213 four groups: mid-/forefoot strike at $10 \mathrm{~km} / \mathrm{h}$ (FF 10), mid-/forefoot strike at $14 \mathrm{~km} / \mathrm{h}$ (FF 14), rearfoot 214 strike at $10 \mathrm{~km} / \mathrm{h}$ (RF 10) and rearfoot strike at $14 \mathrm{~km} / \mathrm{h}$ (RF 14). First, normality was checked with the 215 Shapiro-Wilk test. If data from all groups followed a normal distribution a mixed analysis of variance 216 (ANOVA) was used to determine interaction and main effects (foot strike pattern and running speed) using SPSS v.24 (IBM SPSS, Armonk, New York, USA). Yet, if not all the data in the groups followed a normal distribution, the non-parametric Mann-Whitney $U$ test was performed to compare foot strike pattern differences at 10 and $14 \mathrm{~km} / \mathrm{h}$ separately. To determine the effect of running speed for these datasets, the data was first grouped according to running speed and again checked upon normality. If both datasets were then normally distributed, a paired t-test was performed, if not we performed a Wilcoxon signed-rank test. Statistical significance was considered when $p<0.05$.

\section{Results}

225 Although mean foot strike angle was more than $15^{\circ}$ different between both foot strike groups $(p<$ 226 0.01; Table 2), Triceps Surae metabolic energy consumption was not different between foot strike 227 patterns, regardless of speed or metabolic energy model ( $p>0.35$; Figure 2 ). Moreover, metabolic 228 energy consumed by the individual Triceps Surae muscles, i.e. GM, GL and SOL, was not different 229 between foot strike patterns $(p>0.10)$ independent of the model used or running speed. Furthermore, 230 estimated whole body metabolic energy consumption was not different between foot strike patterns 231 regardless of model or running speed tested $(p>0.14$; Figure 3$)$. As one would expect, running faster 232 resulted in greater metabolic energy consumption in the Triceps Surae muscle group $(p<0.01)$ as well 233 as in all three Triceps Surae individually $(p<0.02)$. Also, whole body metabolic energy consumption 234 was greater when running at $14 \mathrm{~km} / \mathrm{h}$ compared to $10 \mathrm{~km} / \mathrm{h}(\mathrm{p}<0.01)$. 
235 The ratio of metabolic energy consumed by the Triceps Surae relative to whole body metabolic energy 236 consumption ranged between 22 and $32 \%$ across foot strike patterns and running speeds but was not 237 different between foot strike patterns $(p>0.19)$. In contrast, the different models revealed 238 inconsistent results when the effect of speed on this ratio was considered. While U03 and U16 did not 239 show significant differences in this ratio between running speeds ( $p>0.07)$, U10 showed a significant 240 greater ratio at $14 \mathrm{~km} / \mathrm{h}$ compared to $10 \mathrm{~km} / \mathrm{h}(p=0.01)$, whereas B04 showed a significant smaller 241 ratio at $14 \mathrm{~km} / \mathrm{h}$ than at $10 \mathrm{~km} / \mathrm{h}(p=0.02)$. 


\begin{tabular}{|c|c|c|c|c|}
\hline & & Speed & Forefoot strike & Rearfoot strike \\
\hline Foot strike angle & & $10 \mathrm{~km} / \mathrm{h}$ & $-0.4 \pm 4.4$ & $14.8 \pm 3.7$ \\
\hline \multirow[t]{3}{*}{$\left({ }^{o}\right)^{a}$} & & $14 \mathrm{~km} / \mathrm{h}$ & $0.3 \pm 5.3$ & $17.2 \pm 5.4$ \\
\hline & U03 & $10 \mathrm{~km} / \mathrm{h}$ & $26 \pm 4$ & $22 \pm 8$ \\
\hline & & $14 \mathrm{~km} / \mathrm{h}$ & $25 \pm 3$ & $25 \pm 8$ \\
\hline Ratio (\%) & $\mathrm{B} 04^{\mathrm{b}}$ & $10 \mathrm{~km} / \mathrm{h}$ & $26 \pm 3$ & $27 \pm 6$ \\
\hline \multirow{5}{*}{$\left(E_{T S} / E_{W B}\right)$} & & $14 \mathrm{~km} / \mathrm{h}$ & $23 \pm 4$ & $26 \pm 6$ \\
\hline & $\mathrm{U} 10^{\mathrm{b}}$ & $10 \mathrm{~km} / \mathrm{h}$ & $27 \pm 4$ & $28 \pm 9$ \\
\hline & & $14 \mathrm{~km} / \mathrm{h}$ & $28 \pm 5$ & $32 \pm 10$ \\
\hline & U16 & $10 \mathrm{~km} / \mathrm{h}$ & $27 \pm 4$ & $23 \pm 8$ \\
\hline & & $14 \mathrm{~km} / \mathrm{h}$ & $26 \pm 3$ & $26 \pm 9$ \\
\hline
\end{tabular}



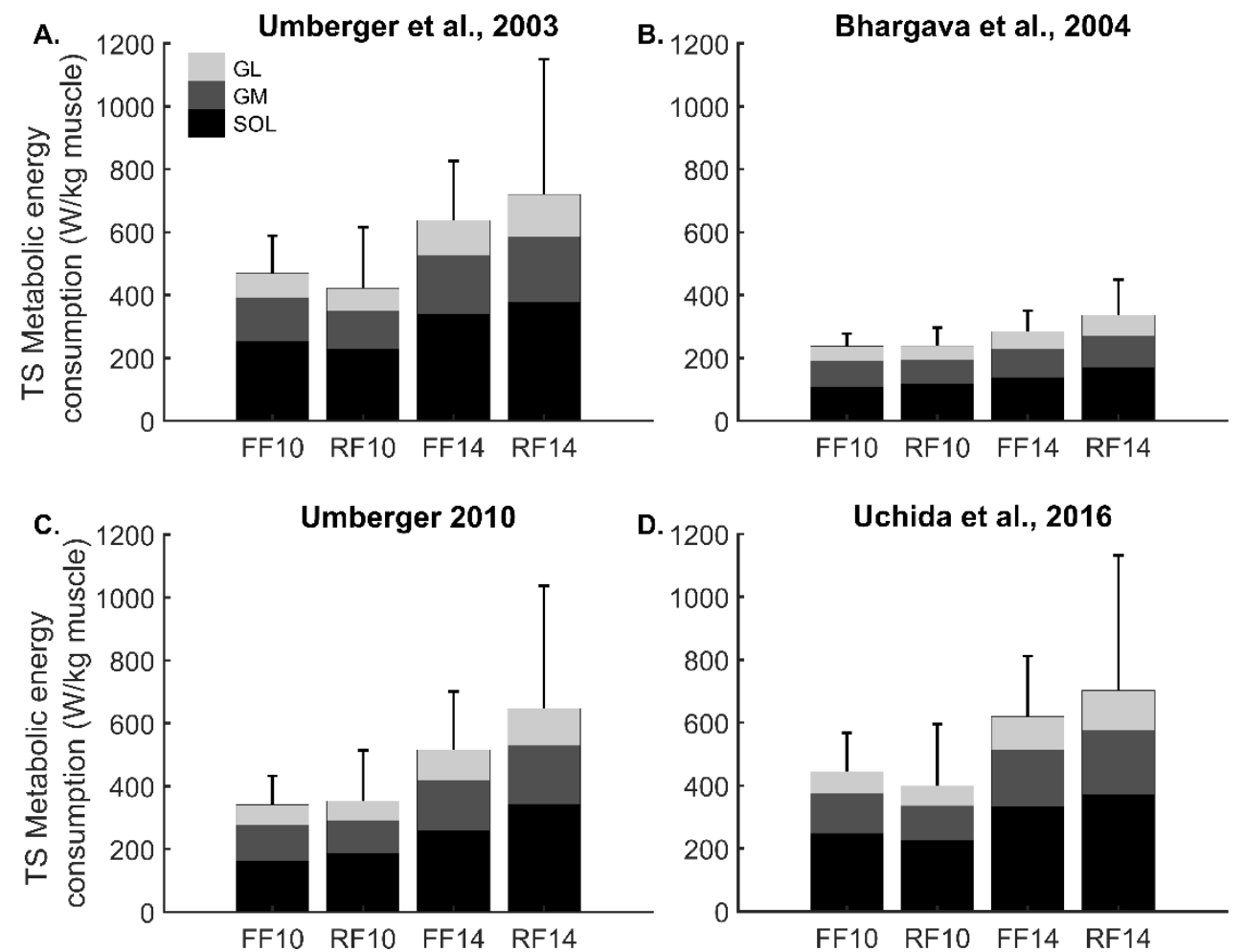

FF10 RF10 FF14 RF14

FF10 RF10 $\quad$ FF14 RF14

Figure 2. Triceps Surae (TS) metabolic energy consumption including individual muscles: Soleus (black), Gastrocnemius medialis (dark grey) and Gastrocnemius lateralis (light grey) in mid-/forefoot strikers (FF, $n=10$ ) and rearfoot strikers (RF, $\boldsymbol{n}=9$ ). Mixed ANOVA or Mann-Whitney $U$ test demonstrated no significant difference in metabolic energy consumed between foot strike patterns, not for individual Triceps Surae muscle $(p>0.10)$ nor for all three muscles together $(p>0.35)$. Mixed ANOVA, paired t-test or Wilcoxon signed-rank test demonstrated significant greater energy consumption at 14km/h compared
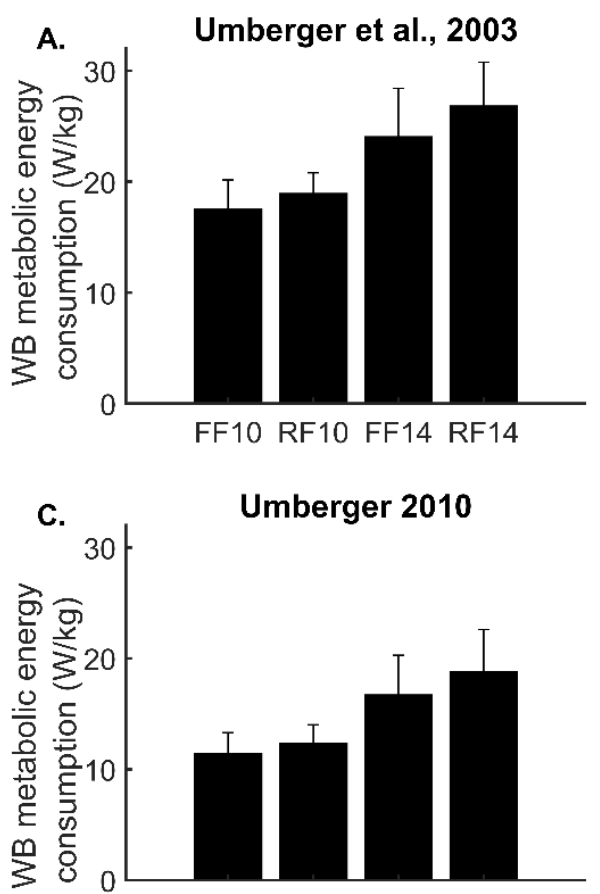

FF10 RF10 FF14 RF14

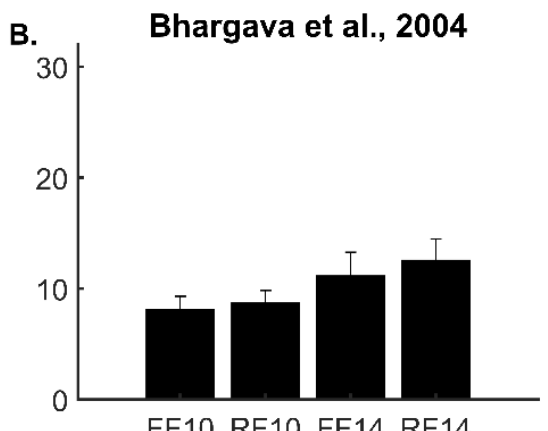

FF10 RF10 FF14 RF14

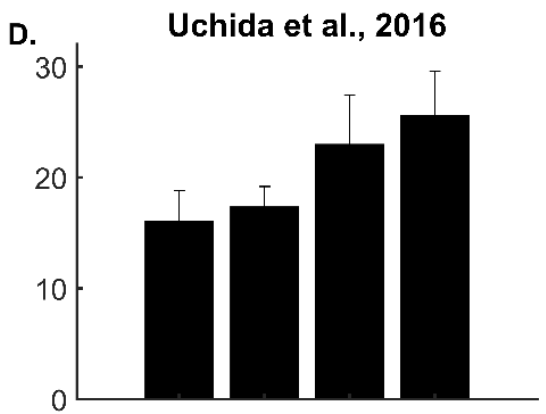

FF10 RF10 FF14 RF14 
Figure 3. Estimated whole body (WB) metabolic energy consumption for all four metabolic energy models used for mid$/$ forefoot strikers at $10 \mathrm{~km} / \mathrm{h}$ (FF10) and $14 \mathrm{~km} / \mathrm{h}$ (FF14) and rearfoot strikers at $10 \mathrm{~km} / \mathrm{h}$ (RF10) and $14 \mathrm{~km} / \mathrm{h}$ (RF14). Mixed ANOVA or Mann-Whitney $U$ test demonstrated no significant difference between foot strike patterns ( $p>0.14)$. Mixed ANOVA, paired t-test or Wilcoxon signed-rank test demonstrated significant increase in energy consumption when running at $14 \mathrm{~km} / \mathrm{h}$ compared to $10 \mathrm{~km} / \mathrm{h}$.

\section{Discussion}

This study investigated the effect of habitual foot strike pattern on simulated Triceps Surae muscle and whole body metabolic energy consumption. We used a dynamic optimization approach in which the Achilles tendon stiffness of the musculoskeletal model was adapted to better match experimental GM ultrasound data (Figure 1). Four different metabolic energy models were incorporated to ensure model independency. In line with our hypothesis, none of the individual Triceps Surae muscles, nor whole body metabolic energy consumption demonstrated significant differences between mid-/forefoot strikers and rearfoot strikers (Figure 2 and Figure 3). Faster running increased both simulated Triceps Surae muscle and whole body metabolic energy consumption. These findings were independent of the metabolic energy model used.

Our results provide additional scientific evidence that mid-/forefoot and rearfoot strike patterns are energetically equivalent. We recently showed that GM muscle force production is greater while muscle contraction velocity is smaller in mid-/forefoot strikers compared to rearfoot strikers, especially during early ground contact (Swinnen et al., 2019). Here, we provide further evidence that the greater muscle forces in mid-/forefoot strikers are more economically produced due to the lower muscle contraction velocities and hence no difference in GM, GL or SOL metabolic energy consumption between foot strike patterns exist. Moreover, previous experimental research already demonstrated that differences in whole body metabolic energy consumption between foot strike patterns are small (Ogueta-Alday et al., 2014) or even non-existing (Cunningham et al., 2010; Gruber et al., 2013; Lussiana et al., 2017; Perl et al., 2012). Studies investigating the effect of gait retraining from rearfoot to forefoot strike running do not find an effect on the metabolic energy consumption during running when enough training sessions ( $\geq 8$ ) were offered (Ekizos et al., 2018; Roper et al., 2017). However, when only two training sessions were provided an initial increase in metabolic cost is reported (Ekizos et al., 2018), indicating the need for habituation. Although it remains possible that more training sessions may further reduce metabolic cost once switched, in general, switching foot strike pattern seems to be ineffective from a performance point of view.

Next to estimated Triceps Surae muscle and whole body metabolic energy rate, the contribution of the Triceps Surae to the whole body metabolic energy rate (i.e. ratio) was also not different between foot strike patterns. However, the effect of running speed was less clear. Two models (U03 and U16) did 
not find a speed effect, while U10 and B04 did find a speed effect, but in opposing directions. With

294 faster running the relative contribution of joint power/work during ground contact seems to gradually 295 shift more towards proximal joints (i.e. hip), especially at running speeds closer to sprinting (Schache 296 et al., 2015). Hence, if a shift in muscle metabolic energy consumption would occur, a shift in the same 297 direction as joint power would have been expected, implying a decreased relative contribution of the 298 Triceps Surae with increasing running speed. However, the difference in running speeds tested in this study was small and our fastest speed did not approach sprinting. Therefore, to better understand the effect of running speed on the distribution of muscle metabolic energy consumption across lower extremity muscles a wider range of running speeds should be investigated. Moreover, by testing a wider range of running speeds, one could also examine whether Triceps Surae metabolic energy consumption between foot strike patterns remains similar outside our tested range of running speeds.

304 Especially faster running speeds would be an interesting future direction as, with faster running, more 305 people tend to mid-/forefoot strike (Breine et al., 2014).

306 Dynamic optimization allowed us to account for muscle-tendon interactions when estimating muscle 307 states. A good match between experimental and predicted muscle states is crucial for good 308 estimations of muscle metabolic energy. We found that it was important to adapt Achilles tendon 309 stiffness to obtain a close match between simulated and measured GM fiber lengths. Using a generic 310 normalized tendon stiffness value of 35 resulted in negligible length changes of the tendinous tissues 311 and as a consequence muscle fascicle length changes were no longer uncoupled from length changes 312 of the entire muscle tendon unit (Fig. S3). Nevertheless, there is ample experimental evidence that the 313 tendinous tissue interacts with the Triceps Surae muscles, uncoupling the muscle fascicle length 314 changes from the length changes of the entire MTU (Fukunaga et al., 2002; Lai et al., 2015; Lichtwark 315 and Wilson, 2008), allowing the muscle fascicles to contract at much slower - more force-efficient 316 velocities implying lower metabolic energy consumption (Hill, 1922; van der Zee, Lemaire and van 317 Soest, 2019). As a result, predicted Triceps Surae muscle metabolic energy consumption with the 318 generic stiff tendon was on average $80 \%$ higher compared to the adapted Achilles tendon stiffness 319 (Fig. S4). Also, estimated whole body metabolic energy consumption was on average $23 \%$ higher 320 compared to the adapted Achilles tendon stiffness (Fig. S5). The discrepancy between the results based 321 on the generic and adapted tendon stiffness values illustrates the importance of a good match 322 between computed and experimental muscle states to obtain reliable results of muscle metabolic 323 energy consumption. Moreover, the increased metabolic energy consumption associated with the stiff 324 tendon emphasizes the importance of the muscle-tendon unit interaction on the metabolic energy 325 consumption during running. 
326 Although our conclusions are independent of the metabolic energy model used, the wide variability in 327 absolute energy rates between the metabolic energy models is remarkable. While B04 and U10 predict 328 experimental whole body metabolic energy consumption rather close to experimental data, whole 329 body metabolic energy consumption predicted by U03 and U16 are almost twice as high as experimentally observed (Batliner et al., 2018; Kipp et al., 2018). The major difference is that U03/U16 neglect eccentric work whereas B04/U10 account for eccentric work. Instead of accounting for negative work, U03/U16 reduce the lengthening heat rate coefficient. Our results (lower energy rates with $\mathrm{U} 03 / \mathrm{U} 16$ ) illustrate that the reduction of the lengthening heat rate more than offsets the exclusion of eccentric muscle work. While we seem to have a good understanding of the energy cost of isometric and concentric muscle contractions, the energy cost during eccentric or stretch-shortening muscle contraction is more debatable. It is clear that eccentric muscle work is more efficiently produced compared to concentric muscle work (Hill, 1960), and therefore it appears reasonable to allow eccentric muscle work and muscle lengthening to reduce the metabolic energy consumption rate of a muscle, however a clear consensus on how to treat eccentric work is still lacking. Also, the energy cost associated with the stretch-shortening of a muscle is still controversial (Holt et al., 2014; van der Zee et al., 2019). Nevertheless, in contrast to the absolute differences, the relative increase in metabolic energy consumption based on all muscle metabolic models when running faster corresponds quite well with the experimental data. Experimental data indicates that increasing the running speed from $10 \mathrm{~km} / \mathrm{h}$ to $14 \mathrm{~km} / \mathrm{h}$ would corresponds with an increase in whole body metabolic energy consumption of approximately 40 to 45\% (Batliner et al., 2018; Kipp et al., 2018). The energy models predict similar increases of $40 \%$ (U03), $41 \%$ (B04), $49 \%$ (U10) and $45 \%$ (U16). In summary, while metabolic energy models do a good job for predicting relative changes, absolute values are not in accordance with experimental data. Therefore, experimental muscle research on how to account for the energy cost of eccentric and stretch-shortening muscle contractions is necessary before recommendations on how to implement these contractions in metabolic energy models can be made.

Our study has some limitations. First, we did not measure Achilles tendon stiffness from our participants and assumed equal normalized Achilles tendon stiffness for all subjects. Kubo et al. (2015) found no difference in Achilles tendon stiffness between foot strike patterns and thus, on average, we can assume equal normalized Achilles tendon stiffness. Mid-/forefoot strikers are reported to earlier activate their Gastrocnemii muscles (Ahn et al., 2014; Swinnen et al., 2019). However due to our optimization criteria (i.e. minimization of muscle activation squared) pre-activation of the Triceps

357 Surae muscles is not predicted. Still, our simulations demonstrate a slightly earlier Triceps Surae 358 muscle activation in mid-/forefoot strikers than rearfoot strikers (Fig. S2). Furthermore, musculoskeletal modelling simplifies human anatomy and functionality. For example, our 
musculoskeletal model lacks a midfoot arch, which has been shown to store and release energy and

361 subsequently reduce the metabolic rate during running (Ker et al., 1987; Stearne et al., 2016). Model

362 parameters describing muscle properties are often derived from cadaver studies and as such may not

363 represent actual muscle properties in healthy men or women. We model the Achilles tendon moment

364 arm as a function of the ankle angle, however Rasske et al. (2017) demonstrated that Achilles tendon

365 moment arm is also load dependent. Moreover, we only took metabolic energy expenditure during

366 ground contact into account, according to Arellano and Kram (2014) only considering ground contact

367 would lead to an underestimation of $7 \%$ of the net metabolic energy expenditure. We used ultrasound

368 data to validate our simulations, a well-known limitation of ultrasound data is that these $2 \mathrm{D}$ images

369 represents a 3D muscle structure, possibly resulting in underestimation of muscle fascicle length

370 changes when there is out of plane muscle movement. Although, we seem to have equally trained

371 runners in both groups (Table 1), we did not collect running performance metrics or whole body

372 metabolic energy consumption and therefore it remains possible that whole body energy consumption

373 or running performance is still different between our groups.

374 In conclusion, we demonstrated that - in contrast with the widespread belief in the running 375 community - none of the foot strike patterns induce a reduction in metabolic energy consumption of 376 the Triceps Surae muscle while running. In agreement with previous experimental research, simulated 377 whole body metabolic energy consumption was also similar between foot strike patterns. Hence, we 378 conclude that none of the foot strike patterns can be associated with a superior running energetics.

379 Yet, we looked into differences in metabolic rate during sub-maximal running, an important 380 performance parameter in distance running. It should be noted that for sprinting energy rate is not as 381 important due to the short distance/time.

\section{Acknowledgements}

383 We thank Li-Ning for providing the running shoes.

\section{Competing interests}

385 No competing interests declared.

\section{Funding}

387 WS is funded by a PhD fellowship from the research foundation Flanders (11E3919N).

\section{References}

Ahn, A. N., Brayton, C., Bhatia, T. and Martin, P. (2014). Muscle activity and kinematics of forefoot 
and rearfoot strike runners. J. Sport Heal. Sci. 3, 102-112.

Altman, A. R. and Davis, I. S. (2012). A kinematic method for footstrike pattern detection in barefoot and shod runners. Gait Posture 35, 298-300.

Arellano, C. J. and Kram, R. (2014). Partitioning the metabolic cost of human running: A task-by-task approach. Integr. Comp. Biol. 54, 1084-1098.

Batliner, M. E., Kipp, S., Grabowski, A. M., Kram, R., Byrnes, W. C., Physiology, I. and States, U. (2018). Does Metabolic Rate Increase Linearly with Running Speed in all Distance Runners? Sport. Med. Int. Open 2, E1-E8.

Bhargava, L. J., Pandy, M. G. and Anderson, F. C. (2004). A phenomenological model for estimating metabolic energy consumption in muscle contraction. J. Biomech. 37, 81-88.

Breine, B., Malcolm, P., Frederick, E. C. and De Clercq, D. (2014). Relationship between running speed and initial foot contact patterns. Med. Sci. Sports Exerc. 46, 1595-1603.

Cavanagh, P. R. and Lafortune, M. A. (1980). Ground reaction forces in distance running. J. Biomech. 13, 397-406.

Cunningham, C. B., Schilling, N., Anders, C. and Carrier, D. R. (2010). The influence of foot posture on the cost of transport in humans. J. Exp. Biol. 213, 790-797.

de Almeida, M. O., Saragiotto, B. T., Yamato, T. P. and Lopes, A. D. (2015). Is the rearfoot pattern the most frequently foot strike pattern among recreational shod distance runners? Phys. Ther. Sport 16, 29-33.

De Groote, F., De Laet, T., Jonkers, I. and De Schutter, J. (2008). Kalman smoothing improves the estimation of joint kinematics and kinetics in marker-based human gait analysis. J. Biomech. 41, 3390-3398.

De Groote, F., Pipeleers, G., Jonkers, I., Demeulenaere, B., Patten, C., Swevers, J. and De Schutter, J. (2009). A physiology based inverse dynamic analysis of human gait: Potential and perspectives. Comput. Methods Biomech. Biomed. Engin. 12, 563-574.

De Groote, F., Kinney, A. L., Rao, A. V. and Fregly, B. J. (2016). Evaluation of Direct Collocation Optimal Control Problem Formulations for Solving the Muscle Redundancy Problem. Ann. Biomed. Eng. 44, 2922-2936.

Ekizos, A., Santuz, A. and Arampatzis, A. (2018). Short- and long-term effects of altered point of ground reaction force application on human running energetics. J. Exp. Biol. 221, jeb176719.

Farris, D. J. and Lichtwark, G. A. (2016). UltraTrack: Software for semi-automated tracking of muscle fascicles in sequences of B-mode ultrasound images. Comput. Methods Programs Biomed. 128, 111-118.

Fletcher, J. R. and MacIntosh, B. R. (2017). Running economy from a muscle energetics perspective. Front. Physiol. 8, 10.3389/fphys.2017.00433.

Fukunaga, T., Kawakami, Y., Kubo, K. and Kanehisa, H. (2002). Muscle and Tendon Interaction During Human Movements. Exerc. Sport Sci. Rev. 30, 106-110.

Gerus, P., Rao, G. and Berton, E. (2015). Ultrasound-based subject-specific parameters improve fascicle behaviour estimation in Hill-type muscle model. Comput. Methods Biomech. Biomed. Engin. 18, 116-123.

Gruber, A. H., Umberger, B. R., Braun, B. and Hamill, J. (2013). Economy and rate of carbohydrate oxidation during running with rearfoot and forefoot strike patterns. J. Appl. Physiol. 115, 194- 
201.

Hamner, S. R., Seth, A. and Delp, S. L. (2010). Muscle contributions to propulsion and support during running. J. Biomech. 43, 2709-2716.

Handsfield, G. G., Meyer, C. H., Hart, J. M., Abel, M. F. and Blemker, S. S. (2014). Relationships of 35 lower limb muscles to height and body mass quantified using MRI. J. Biomech. 47, 631-638.

Hasegawa, H., Yamauchi, T. and Kraemer, W. J. (2007). Foot strike patterns of runners at the 15-km point during an elite-level half marathon. J. Strength Cond. Res. 21, 888-893.

Hill, A. V (1922). The maximum work and mechanical efficiency of human muscles, and their most economical speed. J. Physiol. 56, 19-41.

Hill, A. V. (1960). Production and absorption of work by muscle. Science 131, 897-903.

Holt, N. C., Roberts, T. J. and Askew, G. N. (2014). The energetic benefits of tendon springs in running: is the reduction of muscle work important? J. Exp. Biol. 217, 4365-4371.

Hoogkamer, W., Kipp, S., Spiering, B. A. and Kram, R. (2016). Altered running economy directly translates to altered distance-running performance. Med. Sci. Sports Exerc. 48, 2175-2180.

Jones, A. M. and Carter, H. (2000). The effect of endurance training on parameters of aerobic fitness. Sport. Med. 29, 373-386.

Kasmer, M. E., Liu, X. C., Roberts, K. G. and Valadao, J. M. (2013). Foot-strike pattern and performance in a marathon. Int. J. Sports Physiol. Perform. 8, 286-292.

Ker, R. F., Bennett, M. B., Bibby, S. R., Kester, R. C. and Alexander, R. M. (1987). The spring in the arch of the human foot. Nature 325, 147-149.

Kipp, S., Grabowski, A. M. and Kram, R. (2018). What determines the metabolic cost of human running across a wide range of velocities? J. Exp. Biol. 221, jeb184218.

Kipp, S., Kram, R. and Hoogkamer, W. (2019). Extrapolating metabolic savings in running: Implications for performance predictions. Front. Physiol. 10, 10.3389/fphys.2019.00079.

Koelewijn, A. D., Heinrich, D. and van den Bogert, A. J. (2019). Metabolic cost calculations of gait using musculoskeletal energy models, a comparison study. PLoS One 14, e0222037.

Kubo, K., Miyazaki, D., Tanaka, S., Shimoju, S. and Tsunoda, N. (2015). Relationship between Achilles tendon properties and foot strike patterns in long-distance runners. J. Sports Sci. 33, 665-669.

Lai, A., Lichtwark, G. A., Schache, A. G., Lin, Y.-C., Brown, N. A. T. and Pandy, M. G. (2015). In vivo behavior of the human soleus muscle with increasing walking and running speeds. J. Appl. Physiol. 118, 1266-1275.

Larson, P., Higgins, E., Kaminski, J., Decker, T., Preble, J., Lyons, D., Mclntyre, K. and Normile, A. (2011). Foot strike patterns of recreational and sub-elite runners in a long-distance road race. $J$. Sports Sci. 29, 1665-1673.

Lichtwark, G. A. and Wilson, A. M. (2008). Optimal muscle fascicle length and tendon stiffness for maximising gastrocnemius efficiency during human walking and running. J. Theor. Biol. 252, $662-673$.

Lussiana, T., Gindre, C., Hébert-Losier, K., Sagawa, Y., Gimenez, P. and Mourot, L. (2017). Similar running economy with different running patterns along the aerial-terrestrial continuum. Int. J. Sports Physiol. Perform. 12, 481-489. 
Miller, R. H. (2014). A comparison of muscle energy models for simulating human walking in three dimensions. J. Biomech. 47, 1373-1381.

Moore, I. S. (2016). Is There an Economical Running Technique? A Review of Modifiable Biomechanical Factors Affecting Running Economy. Sport. Med. 46, 793-807.

Ogueta-Alday, A., Rodríguez-Marroyo, J. A. and García-López, J. (2014). Rearfoot striking runners are more economical than midfoot strikers. Med. Sci. Sports Exerc. 46, 580-585.

Patterson, M. A. and Rao, A. V. (2014). GPOPS-II. ACM Trans. Math. Softw. 41, 1-37.

Perl, D. P., Daoud, A. I. and Lieberman, D. E. (2012). Effects of footwear and strike type on running economy. Med. Sci. Sports Exerc. 44, 1335-1343.

Rasske, K., Thelen, D. G. and Franz, J. R. (2017). Variation in the human Achilles tendon moment arm during walking. Comput. Methods Biomech. Biomed. Engin. 20, 201-205.

Roper, J. L., Doerfler, D., Kravitz, L., Dufek, J. S. and Mermier, C. (2017). Gait Retraining from Rearfoot Strike to Forefoot Strike does not change Running Economy. Int. J. Sports Med. 38, 1076-1082.

Schache, A. G., Brown, N. A. T. and Pandy, M. G. (2015). Modulation of work and power by the human lower-limb joints with increasing steady-state locomotion speed. J. Exp. Biol. 218, 24722481.

Stearne, S. M., Alderson, J. A., Green, B. A., Donnelly, C. J. and Rubenson, J. (2014). Joint kinetics in rearfoot versus forefoot running: Implications of switching technique. Med. Sci. Sports Exerc. 46, 1578-1587.

Stearne, S. M., McDonald, K. A., Alderson, J. A., North, I., Oxnard, C. E. and Rubenson, J. (2016). The Foot's Arch and the Energetics of Human Locomotion. Sci. Rep. 6,

Swinnen, W., Hoogkamer, W., Delabastita, T., Aeles, J., De Groote, F. and Vanwanseele, B. (2019). Effect of habitual foot-strike pattern on the gastrocnemius medialis muscle-tendon interaction and muscle force production during running. J. Appl. Physiol. 126, 708-716.

Uchida, T. K., Hicks, J. L., Dembia, C. L. and Delp, S. L. (2016). Stretching your energetic budget: How tendon compliance affects the metabolic cost of running. PLoS One 11, 1-19.

Umberger, B. R. (2010). Stance and swing phase costs in human walking. J. R. Soc. Interface 7, 13291340.

Umberger, B. R., Gerritsen, K. G. M. and Martin, P. E. (2003). A Model of Human Muscle Energy Expenditure. Comput. Methods Biomech. Biomed. Engin. 6, 99-111.

van der Zee, T. J., Lemaire, K. K. and van Soest, A. J. (2019). The metabolic cost of in vivo constant muscle force production at zero net mechanical work. J. Exp. Biol. 222, jeb199158.

Wächter, A. and Biegler, L. T. (2006). On the implementation of an interior-point filter line-search algorithm for large-scale nonlinear programming. Math. Program. 106, 25-57.

Waters, R. L. and Mulroy, S. (1999). The energy expenditure of normal and pathologic gait. Gait Posture 9, 207-231.

Zajac, F. E. (1989). Muscle and tendon: properties, models, scaling, and application to biomechanics and motor control. Crit. Rev. Biomed. Eng. 17, 359-410. 


\section{$391 \quad$ Figure Legends}

392 Figure 1. Simulated (solid) and experimental (dashed) GM muscle fascicle length changes during ground contact in mid393 /forefoot strikers $(A, B ; n=10)$ and rearfoot strikers $(C, D ; n=9)$ at $10 \mathrm{~km} / \mathrm{h}(A, C)$ and $14 \mathrm{~km} / \mathrm{h}(B, D)$. Muscle fascicle length 394 changes are normalized to muscle fascicle length at toe off. Shaded area represent standard deviation.

395 Figure 2. Triceps Surae (TS) metabolic energy consumption including individual muscles: Soleus (black), Gastrocnemius medialis (dark grey) and Gastrocnemius lateralis (light grey) in mid-/forefoot strikers (FF, $n=10$ ) and rearfoot strikers (RF, $n=9)$. Mixed ANOVA or Mann-Whitney $U$ test demonstrated no significant difference in metabolic energy consumed between foot strike patterns, not for individual Triceps Surae muscle $(p>0.10)$ nor for all three muscles together $(p>0.35)$. Mixed ANOVA, paired t-test or Wilcoxon signed-rank test demonstrated significant greater energy consumption at $14 \mathrm{~km} / \mathrm{h}$ compared to $10 \mathrm{~km} / \mathrm{h}(p<0.01)$.

401 Figure 3. Estimated whole body (WB) metabolic energy consumption for all four metabolic energy models used for mid/forefoot strikers at $10 \mathrm{~km} / \mathrm{h}$ (FF10) and $14 \mathrm{~km} / \mathrm{h}$ (FF14) and rearfoot strikers at $10 \mathrm{~km} / \mathrm{h}$ (RF10) and $14 \mathrm{~km} / \mathrm{h}$ (RF14). Mixed ANOVA or Mann-Whitney $U$ test demonstrated no significant difference between foot strike patterns ( $p>0.14)$. Mixed ANOVA,

404 paired t-test or Wilcoxon signed-rank test demonstrated significant increase in energy consumption when running at $14 \mathrm{~km} / \mathrm{h}$

405 compared to $10 \mathrm{~km} / \mathrm{h}$.

406 